Therapie je nach Schweregrad und Risikofaktoren

\title{
Akute Divertikulitis: Meist heilt sie ambulant
}

\author{
Die Divertikulose ist zwar häufig, die Divertikulitis jedoch selten. Und der Verlauf einer Diver- \\ tikulitis ist individuell sehr unterschiedlich. In der Regel führt eine konservative Therapie zur \\ Abheilung, nur selten muss operiert werden.
}

\begin{abstract}
_ Ab dem 50. Lebensjahr finden sich bei fast jedem Zweiten Divertikel im Kolon, wobei vorrangig das Sigma und das Colon descendens betroffen sind. Risikofaktoren sind genetische Prädisposition, Adipositas, ballaststoffarme Ernährung und mangelnde Bewegung. „Doch nur bei weniger als $10 \%$ der Betroffenen kommt es irgendwann zu einer Komplikation, nämlich einer akuten Divertikulitis oder Blutung“, erläuterte Prof. Jörg Ritz, Schwerin. Man nimmt an, dass die Divertikulitis die Folge einer Stuhlimpaktation und einer Obstruktion des Divertikelhalses mit Sekretstau ist. Folge der Druckerhöhung im Divertikel ist eine fokale mukosale Ischämie, die eine bakterielle Invasion in die Mukosa begünstigt.
\end{abstract}

\section{Linksseitige Appendizitis}

Typisch für die akute Divertikulitis ist der meist akut einsetzende Schmerz im linken Unterbauch, der kolikartig oder kontinuierlich empfunden wird. Man spricht auch von einer „linksseitigen Appendizitis“. Dazu kommen häufig Stuhl-

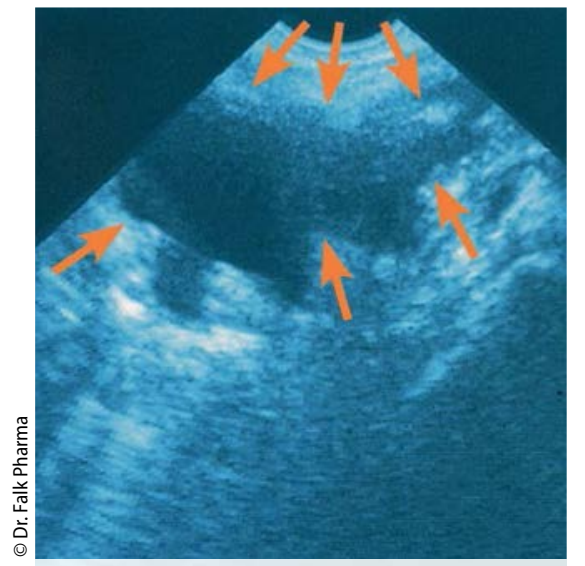

Ultraschall: Abszess bei perforierter Divertikulitis (Pfeile). unregelmäßigkeiten, selten eine rektale Blutung. Die Entzündungsparameter können, müssen aber nicht erhöht sein. Wichtig ist es, mittels bildgebender Diagnostik das Ausmaß der Entzündung zu erfassen, also zu klären, ob eine unkomplizierte oder komplizierte Divertikulitis vorliegt. „Das kann man klinisch nicht zutreffend beurteilen“, so Ritz.

Bildgebendes Verfahren der Wahl ist die Abdomensonografie. Typisch ist die umschriebene Darmwandverdickung i.S. einer Kokarde. Lässt sich sonografisch eine Abszessbildung nicht sicher ausschließen, sollte eine Computertomografie durchgeführt werden. „Die bildgebende Diagnostik ist auch wichtig, um andere Differenzialdiagnosen wie beispielsweise ein Kolonkarzinom ausschließen zu können“, so Ritz. Die Koloskopie ist im Allgemeinen entbehrlich.

\section{Klassifikation nach Schweregrad}

Im Hinblick auf die Therapie werden verschiedene Stadien der Divertikelkrankheit unterschieden:

- Typ 0: asymptomatische Divertikulose

- Typ 1: akute unkomplizierte Divertikulitis ohne (Typ 1a) oder mit phlegmonöser Umgebungsreaktion (Typ 1b)

- Typ 2: akute komplizierte Divertikulitis mit Mikroabszess (Typ 2a), Makroabszess (Typ 2b) oder freie Perforation (Typ 2c)

- Typ 3: chronische rezidivierende oder anhaltende symptomatische Divertikelkrankheit

- Typ 4: Divertikelblutung.

Während die Typen 1 und 2a meist konservativ behandelt werden, besteht bei Typ $2 b$ die Indikation für eine interventionelle Abszessdrainage und bei Typ 2c

\section{Therapie zu Hause}

Eine ambulante orale Antibiotikatherapie der akuten Divertikulitis ist dann möglich, wenn folgende Voraussetzungen erfüllt sind:

- Ultraschall oder CT zeigen eine Divertikulitis ohne Abszess

- Orale Flüssigkeits- und Medikamentenaufnahme sind möglich

- Keine signifikanten Komorbiditäten

- Orale Antibiotika sind verfügbar

- Eine adäquate Schmerzkontrolle ist möglich

- Unterstützung im sozialen Umfeld ist gesichert

- Engmaschige klinische Kontrollen sind möglich.

die dringende Indikation für eine Operation. Bei Typ 3 sollte abhängig von Verlauf und Leidensdruck die elektive Operationsindikation gestellt werden.

\section{Stationär oder nicht?}

In den meisten Fällen ist der Verlauf leicht, nur bei ca. 10\% der Patienten entwickelt sich eine komplizierte Divertikulitis. „Da initial der Verlauf nicht abgesehen werden kann, sind anfänglich engmaschige Kontrolluntersuchungen erforderlich“, so PD Niels Teich, Leipzig. Die Entscheidung für eine stationäre Behandlung sollte sich an der individuellen Risikosituation orientieren. So besteht unter NSAR, Kortikosteroiden und Opiaten sowie bei Rauchern ein erhöhtes Perforationsrisiko, unter ASS und NSAR steigt das Blutungsrisiko. Weitere Indikatoren für einen komplizierten Verlauf sind: Hypertonie, Nierenerkrankungen, Immunsuppression und allergische Disposition.

Dr. Peter Stiefelhagen

- Quelle: Internistenkongress 2016 in Mannheim 